\title{
First case of trans apical implantation of an aortic valve in a patient with dextrocardia
}

\author{
Hellmuth S vH Weich ${ }^{1,4^{*}}$, Jacques van Wyk ${ }^{2}$, Wynand van Zyl ${ }^{3}$, Rocco Vivier ${ }^{2}$, Andre Phillips ${ }^{3}$ and Thomas Mabin ${ }^{3}$
}

\begin{abstract}
We describe the clinical presentation and implantation procedure of the first transcatheter aortic valve implantation described in a patient with dextrocardia.
\end{abstract}

Keywords: Aortic valve, Replacement, Cardiac catheterization/intervention, Heart valve, Transapical, Percutaneous

\section{Background}

Transcatheter aortic valve implantation is a relatively new technology where an aortic valve is implanted via a catheter [usually from the femoral artery]. It may also be implanted via a direct puncture of the left ventricular apex. We describe the first case of transcatheter aortic valve implantation $[\mathrm{TAVI}]$ in a patient with dextrocardia.

\section{Case presentation}

The patient is a 66 year old female known with dextrocardia situs inversus. She also suffers from severe rheumatoid arthritis with multiple joint deformities and vertebral collapse due to steroid use. This has been treated with immunosuppressants over a prolonged period.

She presented to us with New York Heart Association class 3 dyspnoea. Echocardiography confirmed severe aortic stenosis with a mean gradient of $42 \mathrm{mmHg}$ and a valve area of $0.8 \mathrm{~cm}^{2}$. We calculated her logistic EuroSCORE as $15 \%$ but the STS score was $14.5 \%$. She was furthermore turned down for conventional surgery by a cardiothoracic surgeon due to frailty and was referred to our team for a TAVI. Peripheral vessels were diseased and a transapical approach was elected. Preparation of the theatre included switching around the positions of all the operators and theatre tables from our usual configuration: the first operator moved over to the right of the patient and the anaesthetist and anaesthetic machine, cardiologist, second surgeon and echo machine moved to the left of the patient. The case was done in a cath lab

\footnotetext{
* Correspondence: hweich@sun.ac.za

${ }^{1}$ Tygerberg Academic Hospital \& Stellenbosch University, Cape Town, South Africa

Full list of author information is available at the end of the article
}

under general anaesthesia with constant trans esophageal echocardiography [TEE] monitoring. Trans thoracic echocardiography was used to localise the left ventricular apex and a mini-thoracotomy was performed. The apex was pre-closed with a purse string suture and the punctured with a needle. We encountered considerable difficulty crossing the diseased aortic valve and placing the stiff guidewire down into the descending aorta but ascribed this to the unfamiliar angles required to position it [see movie S1]. The valve was predilated without difficulty [see Figure 1]. A $23 \mathrm{~mm}$ Edwards SAPIEN XT ${ }^{\mathrm{TM}}$ valve on an Edwards Ascendra ${ }^{\mathrm{TM}}$ delivery system [Edwards Lifesciences ${ }^{\mathrm{TM}}$, Irvine, CA] was deployed under rapid ventricular pacing [see Figure 2] [see movie S2]. Immediate evaluation with a supra-aortic contrast injection [see Figure 3] as well as TEE confirmed good valve function and minimal paravalvular incompetence. The delivery system was removed and the left ventricular apex closed with the purse string suture. The patient made an unremarkable recovery with discharge from hospital on day 4 post-operative. At her last follow-up 3 months post implantation, she was in NYHA class 1 and the prosthetic valve was functioning well.

\section{Conclusions}

Dextrocardia is a rare condition and when not associated with Kartagener's syndrome, carries a normal life expectancy. It is therefore not unusual for patients to live up to ages where senile aortic stenosis is encountered. TAVI is a new technology requiring new skills for all operators involved and when this procedure is performed on a patient with dextrocardia, even more care should be taken to evaluate each step of the procedure for potential
C Biomed Central

(c) $2012 \mathrm{vH}$ Weich et al; licensee BioMed Central Ltd. This is an Open Access article distributed under the terms of the Creative Commons Attribution License (http://creativecommons.org/licenses/by/2.0), which permits unrestricted use, distribution, and reproduction in any medium, provided the original work is properly cited. 


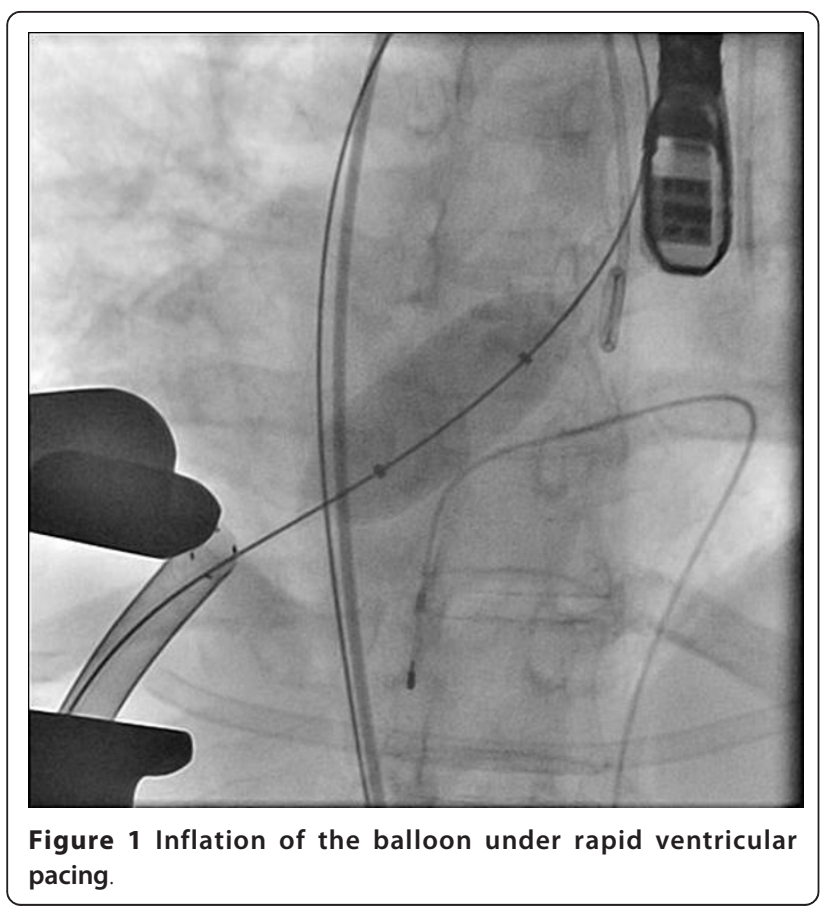

difficulties. We have however shown that with these precautions in place, this procedure is feasible.

\section{Consent}

written informed consent was obtained from the patient prior to the procedure.

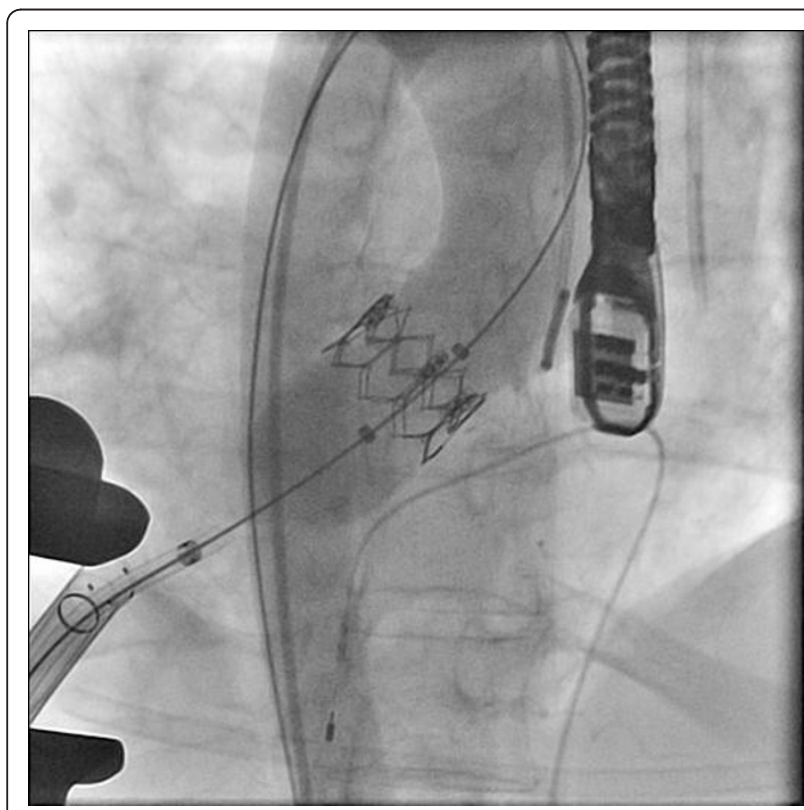

Figure 2 Deployment of the valve.

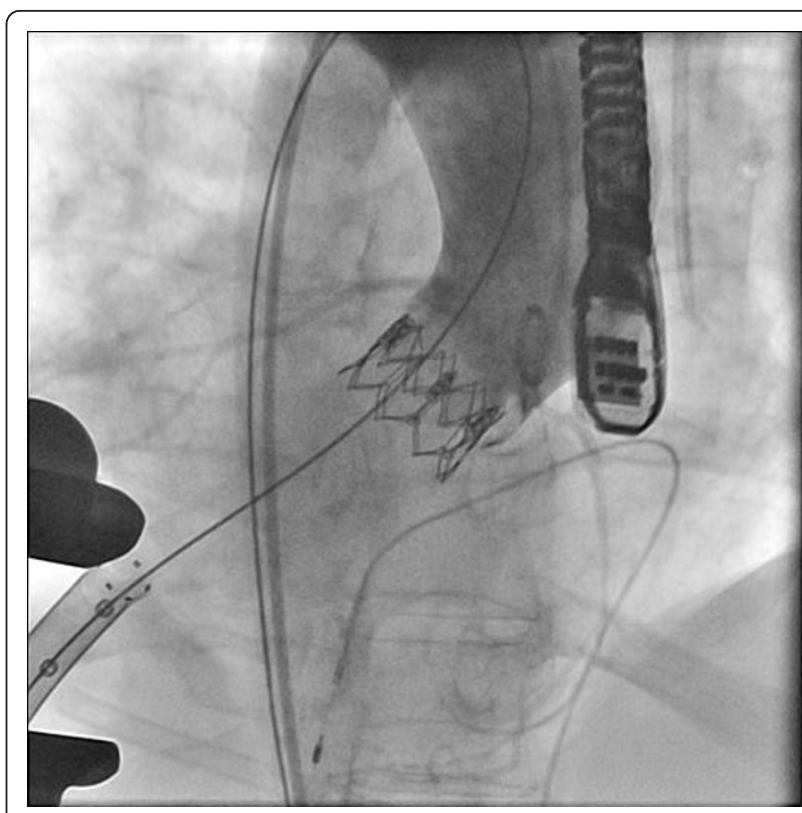

Figure 3 Final result showing good position of the valve and patency of the coronaries.

\section{Additional material}

Additional file 1: Movie S1. This movie shows some of the difficulty we experienced placing the wire down the descending aorta.

Additional file 2: Movie S2. Deployment of the valve. Note that after contrast injection, the position is found to be too high and the device is pulled inferiorly prior to inflation of the balloon. This was done because the patient did not recover well from the first run of rapid pacing and we therefore preferred to reposition and deploy the valve in a single run of rapid pacing.

\section{Author details}

'Tygerberg Academic Hospital \& Stellenbosch University, Cape Town, South Africa. ${ }^{2}$ Panorama Mediclinic, Cape Town, South Africa. ${ }^{3}$ Vergelegen Mediclinic, Cape Town, South Africa. ${ }^{4}$ Division of Cardiology, Department of Internal Medicine, Private bag X1, Tygerberg 7505, South Africa.

\section{Authors' contributions}

$H W, T M, J v W, W v Z, R V \&$ AP all contributed to the pre-operative planning of the case, the changes made to the layout of the theater and performed the procedure. HW acquired the images and wrote the initial manuscript. All authors read and approved the final manuscript

\section{Competing interests}

The authors declare that they have no competing interests.

Received: 28 October 2011 Accepted: 13 March 2012

Published: 13 March 2012

\section{doi:10.1186/1749-8090-7-24}

Cite this article as: $\mathrm{vH}$ Weich et al:: First case of trans apical

implantation of an aortic valve in a patient with dextrocardia. Journal of Cardiothoracic Surgery 2012 7:24. 